\title{
LETTERS
}

\section{Pocket-sized Ultrasound for Physical Diagnosis}

\author{
Benjamin T. Galen, $M D^{7}$ and Daniel J. Schnobrich, $M D^{2}$ \\ 'Department of Internal Medicine, Division of Hospital Medicine, Albert Einstein College of Medicine and Montefiore Medical Center, Bronx, NY, USA; \\ ${ }^{2}$ Department of Medicine, Division of General Internal Medicine, University of Minnesota, Minneapolis, MN, USA.
}

J Gen Intern Med 30(7):883

DOI: $10.1007 / \mathrm{s} 11606-015-3318-2$

(c) Society of General Internal Medicine 2015

\section{$T$ o the editors}

We commend Ojeda et al. for conducting a randomized trial of a pocket-sized ultrasound in the hands of internal medicine residents. ${ }^{1}$ While other studies have shown the feasibility of implementing point-of-care ultrasound curricula for internal medicine residents, the trial by Ojeda et al. had disappointing results. ${ }^{2}$ Several key factors were likely contributory.

One major limitation of this trial was that only $1.5 \mathrm{~h}$ of didactic and $1.5 \mathrm{~h}$ of hands-on training were used in the intervention group. By comparison, emergency medicine residents complete a minimum of $20 \mathrm{~h}$ of didactic training, $80 \mathrm{~h}$ of dedicated ultrasound training, and at least 150 supervised ultrasound scans prior to being assessed for competency. ${ }^{3}$

In the trial by Ojeda et al., the pathology of the study participants was particularly limited. ${ }^{1}$ The cardiac conditions listed in Table 1 of the article included structural heart disease and many valvular abnormalities, but pericardial effusion and left ventricular systolic dysfunction were not included. This choice is puzzling, since clinicians with limited training in point-of-care ultrasound have previously been shown to detect left ventricular systolic dysfunction and pericardial effusions, but not valvular heart disease. ${ }^{4}$

Of the 17 physical exam abnormalities listed in Table 1, only three were abdominal pathologies (hepatomegaly, splenomegaly, and ascites). ${ }^{1}$ The authors stratified their data by "organ system," but did not provide results for each specific abnormality. Assessing for organomegaly might be a more advanced skill than the binary question of whether ascites is present or absent; the reader is left wondering if the intervention group detected ascites reliably even if organomegaly was not well diagnosed.
The intervention group in the study by Ojeda et al. learned chest ultrasound techniques from attending radiologists, but they weren't tested on them: the ten study subjects did not exhibit common pulmonary pathology (other than one patient with a paralyzed left hemidiaphragm). ${ }^{1}$ Diagnosing pulmonary edema, pneumothorax, and pleural effusions by point-ofcare ultrasound is standard practice in emergency medicine, critical care, and pulmonology. ${ }^{5}$

Further studies are needed to determine what point-of-care ultrasound skills should be taught to internal medicine residents. The trial by Ojeda et al. shows that $3 \mathrm{~h}$ of ultrasound training is insufficient to improve residents' bedside diagnostic skills (primarily for valvular heart disease), but additional conclusions about the potential for point-of-care ultrasound as an adjunct to the traditional physical examination cannot be made from this data.

Conflict of interest: All authors contributed to the preparation of this manuscript. We have no conflicts of interest to disclose or any financial disclosures to report.

Corresponding Author: Benjamin T. Galen, MD; Department of Internal Medicine, Division of Hospital Medicine, Albert Einstein College of Medicine and Montefiore Medical Center, Suite 2-76 1825 Eastchester Road, Bronx, NY 10461, USA (e-mail: bgalen@montefiore.org).

\section{REFERENCES}

1. Ojeda JC, Colbert JA, Lin X, et al. Pocket-sized ultrasound as an aid to physical diagnosis for internal medicine residents: a randomized trial. J Gen Intern Med. 2015;30(2):199-206. doi:10.1007/s11606-014-3086-4.

2. Schnobrich DJ, Olson AP, Broccard A, et al. Feasibility and acceptability of a structured curriculum in teaching procedural and basic diagnostic ultrasound skills to internal medicine residents. J Grad Med Educ. 2013;5(3):493-7. doi:10.4300/JGME-D-12-00214.1.

3. American College of Emergency Physicians Policy Statement, Emergency Ultrasound Guidelines. 2008. Available at: http://www.acep.org/ClinicalPractice-Management/Ultrasound/. Accessed 28 Feb 2015

4. Martin LD, Howell EE, Ziegelstein RC, et al. Hand-carried ultrasound performed by hospitalists: does it improve the cardiac physical examination? Am J Med. 2009;122(1):35-41. doi:10.1016/j.amjmed.2008.07.022.

5. Moore CL, Copel JA. Point-of-care ultrasonography. N Engl J Med. 2011;364(8):749-57. doi:10.1056/NEJMra0909487. 